\title{
Light exposure of oocytes and pregnancy rates after their transfer in the rabbit
}

\author{
J. M. Bedford*† and A. Dobrenis $\dagger$ \\ Departments of $\dagger$ Obstetrics and Gynecology and of *Cell Biology and Anatomy, \\ Cornell University Medical College, New York, NY 10021, USA
}

\begin{abstract}
Summary. Groups of unfertilized and pronuclear stage rabbit oocytes were exposed to fluorescent light of $3250 \mathrm{~lx}$ for $20-30 \mathrm{~min}$ at $37^{\circ} \mathrm{C}$. In 6 experiments with fertilization achieved in vivo, 54\% of the zygotes exposed as secondary oocytes and $67 \%$ of lightprotected controls had implanted and developed normally 16 days after transfer to the contralateral oviducts of synchronized recipients. When pronuclear oocytes were exposed similarly in 8 experiments, $63 \%$ had established a normal pregnancy at 16 days after transfer compared to $65 \%$ of the controls. In 5 of these pregnancies which were allowed to proceed to term, all the young born appeared normal.

Though similar in size, it is not clear whether the rabbit oocyte constitutes a suitable model for the human oocyte in regard to the effects of visible light. However, the level of exposure used here is 200-300 times that experienced during normal in-vitro manipulation of human eggs. The absence of significant effects should allay concerns that light is a negative factor in the normal procedure of in-vitro fertilization in man.
\end{abstract}

Keywords: oocytes; zygotes; light exposure; in-vitro fertilization; pregnancy rate; rabbit

\section{Introduction}

In the course of experimental manipulation, or during in-vitro fertilization, oocytes and early zygotes are exposed to daylight or to artificial light for variable periods. This has no consistent inhibitory effect on their potential since many eggs fertilized in vitro develop normally. On the other hand, there is a significant failure to implant after transfer of zygotes and early embryos, and it has seemed possible that lesions caused by light exposure might in some instances contribute to that failure. For instance, a negative effect of light on the in-vitro cleavage and cytoplasmic organization of rabbit zygotes has been reported (Daniel, 1964; Schumacher \& Fischer, 1988; Hegele-Hartung et $a l ., 1988$ ) and prior light exposure has been shown to result in a significant incidence of chromosomal and pronuclear aberrations in hamster oocytes following fertilization in vitro (Hirao \& Yanagimachi, 1978). This outcome in hamster oocytes was attributed to anomalies of the second meiotic division. While light has been reported to cause single strand breaks and protein-DNA crosslinking in cultured somatic cells (Bradley et al., 1978; Gantt et al., 1979) nothing appears to be known of the effects of visible light on oocyte DNA.

In recent years, the possibility of light-related effects has been mentioned in connection with invitro fertilization of human eggs (e.g. Jones et al., 1984; Schumacher \& Fischer, 1988). The results of Hirao \& Yanagimachi (1978) especially seemed to provide some basis for concern to an extent that we incorporated red lights into the design of our own in-vitro fertilization laboratory. It is of course, difficult to examine this issue directly using human oocytes. Rabbit oocytes may not necessarily provide a model that allows a simple extrapolation of results to man. At least, however, they are closely similar in size to human oocytes. Therefore, we have examined the effects of light exposure, cumulatively 200-300 times greater than human eggs or zygotes experience in our 
in-vitro fertilization procedure, on the ability of unfertilized and pronuclear rabbit oocytes to implant and develop as normal embryos.

\section{Materials and Methods}

Light exposure of secondary oocytes. For each of 6 experiments, 2 post-pubertal New Zealand White female rabbits were either superovulated by s.c. injection of pig follicle-stimulating hormone (FSH; Sigma, St Louis, MO, USA), 0.5 i.u./day for 3 days, and finally one injection of human chorionic gonadotrophin (hCG; Sigma) 50 i.u., according to the protocol of Kennelly \& Foote (1965), or were given only an ovulation injection of 50 i.u. hCG. The rabbits were killed by cervical dislocation, $14 \mathrm{~h}$ after hCG, and unfertilized oocytes were flushed from the oviducts with phosphatebuffered saline (PBS) containing $20 \%$ heated rabbit serum, crystalline penicillin $(60 \mathrm{mg} / \mathrm{l})$ and $0.05 \%$ hyaluronidase (bovine type IV; Sigma). This step involved their exposure to dim light for approximately 30 sec. The eggs, invested then primarily by corona radiata, were identified in the flush using a dissecting microscope equipped with a red filter. Individual oocytes were pooled and divided into two groups, each group being placed in separate 35-mm Petri dishes (Falcon: American Scientific, Raritan, NJ, USA) containing PBS + 20\% homologous serum.

While one egg dish remained exposed, the other was completely covered with aluminium foil, thus preventing any exposure of the control oocytes to light. Both dishes were then subjected at $37^{\circ} \mathrm{C}$ to a fluorescent source $(2 \times$ General Electric $40 \mathrm{CW}$ Cool White tubes) giving emissions in the $350-740 \mathrm{~nm}$ range, the spectrum being closely similar to that shown in Fig. 1 of Schumacher \& Fischer (1988). At the distance employed, this source provided a measured illumination intensity of $3250 \mathrm{~lx}$. After exposure for $20-30 \mathrm{~min}$ at that intensity, the light-exposed and light-protected oocytes were transferred as separate groups via flank incisions to one or other of the oviducts of pentobarbitoneanaesthetized females. These had been inseminated intratubally $11 \mathrm{~h}$ previously via flank incisions with $\sim 1 \times 10^{6}$ washed ejaculated rabbit spermatozoa/per tube. The light-exposed and -protected oocytes were recovered 7-9 h later by flushing the respective oviducts, and were examined for fertilization, as indicated by pronucleus formation. At the high-power magnification of the dissecting microscope this is easily determined by the experienced observer, and is characterized in rabbit eggs by a doughnut appearance with a central light-coloured area of cytoplasm. In 6 experiments, using 1 or 2 recipients according to the zygote number available, groups of the light-exposed and -protected pronuclear stage eggs (3-7 per oviduct depending on the number available in each experiment) were then transferred as separate groups to one or other oviduct of a pseudopregnant recipient, synchronized by injection of 50 i.u. hCG $24 \mathrm{~h}$ previously. After closure of the flank incisions and i.m. administration of penicillin (Wycillin) the zygote recipients were left until the 16th day of pregnancy. They were then killed by cervical dislocation, the ovaries were examined for corpora lutea and each uterine horn for implantation sites. Finally, the normality of the developing embryo in each implantation site was evaluated visually.

Light exposure of pronuclear eggs. In each of 8 separate experiments, 2 does were induced to superovulate with FSH and hCG, as described above. At the time of hCG injection, these egg donors were mated twice with fertile males, or, in the absence of receptivity, were artificially inseminated per vaginam with ejaculated spermatozoa collected with an artificial vagina. The females were killed by cervical dislocation $18-20 \mathrm{~h}$ later, a time when DNA replication would have been completed in most zygotes (Oprescu \& Thibault, 1965; Szollosi, 1966). The oviducts were fiushed with PBS $+20 \%$ serum, and the eggs were identified in dim light with a red filter on the stage of the dissecting microscope. About half of the fertilized (pronuclear) eggs, selected as described above and kept in 35-mm Petri dishes in PBS $+20 \%$ serum at $37^{\circ} \mathrm{C}$, were then exposed to a fluorescent light source as in the previous experiment. Control zygotes were treated similarly except that the Petri dishes were covered with foil to exclude all light. Equal groups of 6 or 7 light-exposed or -protected zygotes were then transferred as separate groups via flank incisions into the ampulla of one or other oviduct of hCG-synchronized recipients. In this series, the uterine horns of the recipients were examined for implantation sites at 16 days and again at about 27 days of the pregnancy, via a mid-line laparotomy. In 5 of the experiments the females were allowed to go to term and the live-born young were assessed for normal development.

The results of both series are given in the form of raw data. These data were analysed for significant differences between experimental and control groups using the Wilcoxon signed rank test.

\section{Results}

When eggs exposed to light as unfertilized oocytes were transferred to synchronized recipients at the pronuclear stage after fertilization in vivo, a similar number of normal uterine implantation sites was present at 16 days on the side receiving the light-exposed group as on that receiving the lightprotected (control) zygotes (Table 1). A low number of resorption sites was present on each side. No developmental abnormalities were observed in any of the fetuses. Using the Wilcoxon signed rank test there was no significant difference between the light-exposed and dark controls at the 0.05 
level. However, while no effects of light on rabbit oocytes were revealed even at the excessive intensity employed, it should be noted that, with 35-37 eggs per group, occasional effects might go undetected.

Table 1. Pregnancy rates* after tubal transfer to synchronized foster recipients of pronuclear rabbit zygotes exposed to light as secondary oocytes

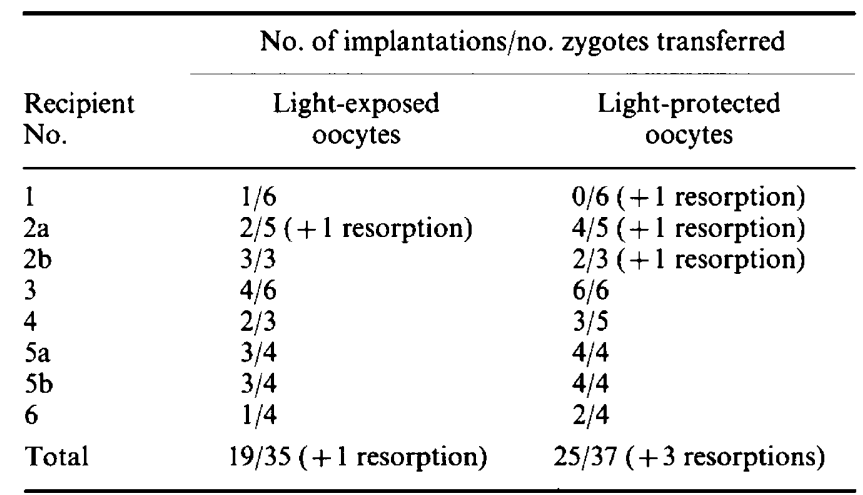

*Evaluated on Day 16.

$95 \%$ confidence interval for the difference in implantation rates including resorptions: $-2 \cdot 8 \%$ to $+40 \cdot 8 \%$.

When oocytes fertilized in vivo were exposed for 20-30 min to fluorescent light soon after fertilization at the pronuclear stage, again a similar proportion of the experimental and control (nonexposed) zygotes implanted and developed normally after transfer, with no significant differences between the groups (Table 2). In this series of 8 experiments, there were no resorptions at 16 days.

Table 2. Pregnancy rates after tubal transfer to synchronized foster recipients of rabbit zygotes immediately after light exposure at the pronuclear stage

\begin{tabular}{lcc}
\hline & \multicolumn{2}{c}{ No. of implantations/ no. transferred } \\
\cline { 2 - 3 } Recipient & $\begin{array}{c}\text { Light-exposed } \\
\text { zygotes }\end{array}$ & $\begin{array}{c}\text { Light-protected } \\
\text { zygotes }\end{array}$ \\
\hline 7 & $4 / 7^{*}(4) \dagger$ & $5 / 7(4)$ \\
8 & $0 / 7(0)$ & $3 / 7(3)$ \\
9 & $7 / 7(1)$ & $7 / 7(0)$ \\
10 & $5 / 6(4)$ & $5 / 6(5)$ \\
11 & $5 / 6(5)$ & $5 / 6(5)$ \\
12 & $5 / 6(1)$ & $4 / 6(3)$ \\
13 & $4 / 6$ & $4 / 6$ \\
14 & $2 / 6$ & $0 / 6$ \\
Total & $32 / 51$ & $33 / 51$ \\
\hline
\end{tabular}

*Normal implantation sites evaluated on Day 16.

†Normal implantation sites evaluated on Day 27.

$95 \%$ confidence interval for the difference in implantation rates: $-17 \%$ to $+21 \%$. 
However, in 6 recipients allowed to proceed further, some losses occurred between the 16-day and a second 27-day laparotomy examination, but equally so in the experimental and control populations, indicating a probable effect of the mid-term laparotomy. The young born alive in the 5 pregnancies allowed to proceed to term obviously could not be attributed to one or another group, but all these young appeared normal.

\section{Discussion}

Results of controlled embryo transfer studies in the rat (Shalgi, 1984) suggested that the in-vitro fertilization procedure per se has some negative element for establishment of a normal pregnancy, but the basis for this was not clear. Further studies using rabbits and rats (Al-Hasani et al., 1986; Vanderhyden et al., 1986; Vanderhyden \& Armstrong, 1988) indicate that in-vitro fertilization may lead to some slowing of the rate of development of the early embryo and so to a possible asynchrony between it and the maternal environment. It has seemed entirely reasonable also that one factor contributing to problems with in-vitro fertilization may be a damaging random effect of exposure to natural or artificial light, in view of the meiotic anomalies it created in hamster oocytes (Hirao \& Yanagimachi, 1978) and of reports of effects on the cytoplasm and on thymidine incorporation after light exposure of rabbit zygotes (Hegele-Hartung et al., 1988; Schumacher \& Fischer, 1988). It is therefore important to note the absence here of any detectable effect of relatively prolonged intensive light exposure of unfertilized rabbit oocytes or of pronuclear oocytes on their subsequent ability to implant and develop normally to mid-pregnancy.

The present experiments were designed to allow fertilization to occur in vivo. Thus, aside from the transfer procedure, the prime variable was the light-exposure step. As noted, the group size was rather small for detection of very minor effects. However, we emphasize that the time for which the eggs were exposed to light $(20-30 \mathrm{~min})$ was much in excess of that experienced during normal in-vitro fertilization or other common manipulation procedures. Moreover, the intensity of the exposure (3250 lx) was significantly greater than that found to disturb hamster oocyte meiosis (Hirao \& Yanagimachi, 1978), or that prevailing during in-vitro fertilization of human oocytes. In fact, we calculate that the cumulative exposure here (i.e. light intensity $\times$ time) was as much as 300 times that experienced by human eggs and zygotes in our own in-vitro fertilization laboratory, in which the lighting nevertheless allows easy observation at every manipulation step.

Expected losses did occur in our experimental and in our control embryo populations, but to an equivalent degree in both. The normal pregnancy rate was $\sim 63 \%$ after transfer of rabbit eggs exposed at the pronuclear stage, and $54 \%$ developed normally after transfer of zygotes previously exposed as secondary oocytes. These results appear comparable or marginally better than those in most embryo transfer studies after in-vivo fertilization in rabbits, (Chang, 1948); Maurer et al., 1968; Overstreet \& Bedford, 1976; Binkerd \& Anderson, 1979) even though the eggs shown in Table 1 were in fact subject to two transfer steps.

This study suggests that rabbit eggs are much less vulnerable to light than are those of the golden hamster. It is possible that the apparent sensitivity of the hamster oocyte to visible light could be an idiosyncratic phenomenon, perhaps related in part to its small size. For this reason it would be prudent now to examine whether light also adversely affects the eggs of the rat and other rodents used for in-vitro studies.

The rabbit oocyte may not be a good model for the effects of light on the human oocyte because there are some obvious differences. With the stereo dissecting microscope, pronuclei are much more easily seen before syngamy in human than in rabbit eggs, perhaps a reflection of organelle density and/or distribution, or possible of oocyte pigmentation. After fertilization rabbit zygotes cleave into 2 and then 4 cells significantly earlier than do human zygotes. It is clear also that timing of expression of the embryonic genome can vary quite widely among different mammalian species (Manes, 1973; Bolton et al., 1984; Crosby et al., 1988). It therefore cannot be assumed that 
similarity in oocyte size necessarily provides grounds from which to extrapolate directly the present findings in rabbits to the human situation.

On the other hand, the exposure involved here should allay concerns that light has any significant negative impact on in-vitro fertilization pregnancy rates when normal procedures are in use. The light exposure in the present experiments does approximate that bringing a first significant $(P<0.05)$ reduction of thymidine incorporation by rabbit zygotes in the study of Schumacher \& Fischer (1988), who suggested that exposure to light could contribute to the low rate of pregnancies that follow human in-vitro fertilization procedures. We stress, however, that not only were there no detectable effects on rabbit embryo development and survival in the present instance, but, as noted above, the cumulative light exposure was 200-300 times more than human oocytes and zygotes experience during normal in-vitro procedures. Intuitively, it seems sensible that a situation involving prolonged intensive light exposure should be avoided. It would appear unnecessary, however, to compromise the efficiency of the in-vitro fertilization procedure in order to minimize light exposure of eggs and early embryos.

Supported by a grant from the Calder Foundation.

\section{References}

Al-Hasani, S., Trotnow, S., Sadtler, C. \& Hahn, J. (1986) In-vitro fertilization and embryo transfer of preovulatory rabbit oocytes. Eur. J. Obstet. Gynecol. Reprod. Biol. 21, 187-195.

Binkerd, P.E. \& Anderson, G.B. (1979) Transfer of cultured rabbit embryos. Gamete Res. 2, 65-73.

Bradley, M.O., Erickson, L.C. \& Kohn, K.W. (1978) Non-enzymatic DNA strand breaks induced in mammalian cells by fluorescent light. Biochem. Biophys. Acta 520, 11-20.

Bolton, V.N., Oades, P.J. \& Johnson, M.H. (1984) The relationship between cleavage, DNA replication and gene expression in the mouse 2-cell embryo. $J$. Embryol. exp. Morph. 79, 139-163.

Chang M.C. (1948) Transplantation of fertilized rabbit ova: the effect on viability of age, in-vitro storage period, and storage temperature. Nature, Lond. 161, 978-979.

Crosby, I.M., Gandolfi, F. \& Moor, R.M. (1988) Control of protein synthesis during early cleavage of sheep embryos. J. Reprod. Fert. 82, 769-775.

Daniel J.C. (1964) Cleavage of mammalian ova inhibited by visible light. Nature, Lond. 201, 316-317.

Gantt, R., Jones G.M., Stephens, E.V., Baeck, A.E. \& Sanford, K.K. (1979) Visible light-induced DNA crosslinks in cultured mouse and human cells. Biochem. Biophys. Acta 565, 231-240.

Hegele-Hartung, C., Schumacher, A. \& Fischer, B. (1988) Ultrastructure of preimplantation rabbit embryos exposed to visible light and room temperature. Anat. Embryol. 178, 229-241.

Hirao, Y. \& Yanagimachi, R. (1978) Detrimental effect of visible light on meiosis of mammalian eggs in vitro. $J$. exp. Zool. 206, 365-369.

Jones, H.W., Acosta, A.A., Andres, M.C., Garcia, J.E., Jones, G.S., Mayer, J., McDowell, J.S., Rosenwaks, Z., Sandow, B.A., Veeck, L.L. \& Wilkes, C.A. (1984) Three years of in vitro fertilization at Norfolk. Fert. Steril. 42, 826-834.
Kennelly, J.J. \& Foote, R.H. (1965) Superovulatory response of pre- and post-pubertal rabbits to commercially available gonadotrophins. J. Reprod. Fert. 9, 177-188.

Manes, C. (1973) The participation of the embryonic genome during early cleavage in the rabbit. Devl Biol. 32, 453-459.

Maurer, R.R., Hunt, W.L., Van Vleck, L.D. \& Foote, R.H. (1968) Developmental potential of superovulated rabbit ova. J. Reprod. Fert. 15, 171-175.

Oprescu, St. \& Thibault, C. (1965) Time and duration of DNA synthesis in rabbit eggs after sperm penetration. Annls Biol. anim. Biochim. Biophys. 5, 151-156.

Overstreet, J.W. \& Bedford, J.M. (1976) Embryonic mortality in the rabbit is not increased after fertilization by young epididymal spermatozoa. Biol. Reprod. 15, 54-57.

Schumacher, A. \& Fischer, B. (1988) Influence of visible light and room temperature on cell proliferation in preimplantation rabbit embryos. J. Reprod. Fert. 84, 197-204.

Shalgi, R. (1984) Developmental capacity of rat embryos produced by in-vivo or in-vitro fertilization. Gamete Res. 10, 77-82.

Szollosi, D. (1966) Time and duration of DNA synthesis in rabbit eggs after sperm penetration. Anat. Rec. 154, 209-212.

Vanderhyden, B.C. \& Armstrong, D.T. (1988) Decreased embryonic survival of in-vitro fertilized oocytes in rats is due to retardation of preimplantation development. J. Reprod. Fert. 83, 851-857.

Vanderhyden, B.C., Rouleau, A., Walton, E.A. \& Armstrong, D.T. (1986) Increased mortality during early embryonic development after in-vitro fertilization of rat oocytes. J. Reprod. Fert. 77, 401-409. 